\title{
Experimental estimation of one-parameter qubit gates in the presence of phase diffusion
}

\author{
Davide Brivio, Simone Cialdi, and Stefano Vezzoli \\ Dipartimento di Fisica dell'Università degli Studi di Milano, I-20133 Milano, Italia and \\ INFN, Sezione di Milano, I-20133 Milano, Italia \\ Berihu Teklu Gebrehiwot, Marco G. Genoni, and Stefano Olivares \\ CNISM UdR Milano Università, I-20133 Milano, Italia and \\ Dipartimento di Fisica dell'Università degli Studi di Milano, I-20133 Milano, Italia \\ Matteo G. A. Paris \\ Dipartimento di Fisica dell'Università degli Studi di Milano, I-20133 Milano, Italia, \\ CNISM UdR Milano Università, I-20133 Milano, Italia, and \\ ISI Foundation, I-10133 Torino, Italia \\ (Received 9 October 2009; published 7 January 2010)
}

\begin{abstract}
We address estimation of one-parameter qubit gates in the presence of phase diffusion. We evaluate the ultimate quantum limits to precision, seek optimal probes and measurements, and demonstrate an optimal estimation scheme for polarization encoded optical qubits. An adaptive method to achieve optimal estimation in any working regime is also analyzed in detail and experimentally implemented.
\end{abstract}

DOI: 10.1103/PhysRevA.81.012305

PACS number(s): 03.67.Lx, 03.65.Wj, 42.50.St

\section{INTRODUCTION}

Suppose you are given a black box which operates on qubits. The reconstruction of the corresponding quantum operations [1-3] is critical to verify its actions as a quantum logic gate [4] as well as to characterize decoherence processes [5]. Let us consider the case when the action of the device is described by the unitary operator $U_{\phi}=\exp \left\{-i \sigma_{n} \phi\right\}$ and corresponds to a phase-shift (rotation), $\phi$, about a known axis, $n$. This is the simplest operation on a qubit and realizes a one-parameter logical gate, which allows, combined with the Hadamard gate, the transformation of any qubit state into another. The quantum characterization [6-12] of this kind of device is of interest for quantum information processing and amounts to estimating the phase shift by measuring a suitable observable at the output. In the following, we fix the reference frame and assume, without loss of generality, rotations $U_{\phi}=\exp \left\{-i \sigma_{z} \phi\right\}$ about the $z$ axis.

Under ideal conditions the estimation of the phase-shift consists of preparing a qubit in a known pure state, $\varrho=$ $\left|\psi_{0}\right\rangle\left\langle\psi_{0}\right|$, and then performing suitable measurements on the (pure) shifted state $\varrho_{\phi}=U_{\phi} \varrho U_{\phi}^{\dagger}$. In a realistic implementation, however, the propagation of a qubit is unavoidably accompanied by some noise, which influences the estimation scheme and usually degrades the overall precision. In this article we address estimation in the presence of the most detrimental kind of noise for a phase gate, that is, nondissipative phase noise, which destroys the off-diagonal elements of the density matrix and thus the information on the imposed phase shift. We evaluate the ultimate quantum limits to precision in the presence of noise and determine both the optimal preparation of the probe qubit and the optimal measurement to be performed at the output. The optimal estimation scheme is then experimentally demonstrated for polarization encoded optical qubits, together with an adaptive method to achieve optimal estimation in any working regime.
This article is structured as follows. In Sec. II we describe the system under investigation focusing on the ultimate limits to precision of phase-shift estimation in the presence of phase noise. The quantum Cramér-Rao limit as well as the optimal quantum estimator are explicitly given. Section III introduces a realistic scenario for qubit phase-shift estimation: an estimation scheme based on spin measurements is described in detail and the corresponding Fisher information is derived. In Sec. IV we turn our attention to optical qubit systems and describe phase-shift estimation under two different approaches: the inversion method and the Bayesian estimation. We also investigate numerically the robustness of the Bayesian analysis with respect to the inversion method in the nonasymptotic regime, that is, in the case of small amounts of data. Section $\mathrm{V}$ is devoted to the experimental demonstration of the optimal scheme for phase-shift estimation and of the adaptive method to achieve the quantum Cramér-Rao bound in any working regime. Section VI closes the article with some concluding remarks.

\section{PHASE-SHIFT ESTIMATION IN QUBIT SYSTEMS}

The measurement scheme we are going to address is schematically depicted in Fig. 1. One has a single qubit, initially prepared in the pure state $\varrho=\left|\psi_{0}\right\rangle\left\langle\psi_{0}\right|$, that undergoes an unknown phase-shift $\phi$ imposed by the unitary operator $U_{\phi}$. Before being measured the shifted state $\varrho_{\phi}=U_{\phi} \varrho U_{\phi}^{\dagger}$ is degraded by a nondissipative phase noise occurring during the propagation. The effect of this kind of noise on the qubit density matrix, given a noise factor $\gamma$, can be described by the following master equation (ME):

$$
\dot{\varrho}_{\phi, \Delta^{2}}=\gamma \mathcal{L}\left[\sigma_{+} \sigma_{-}\right] \varrho_{\phi, \Delta^{2}},
$$

where $\mathcal{L}[A] \varrho_{\phi, \Delta^{2}}=\frac{1}{2}\left\{\left[A \varrho_{\phi, \Delta^{2}}, A^{\dagger}\right]+\left[A, \varrho_{\phi, \Delta^{2}} A^{\dagger}\right]\right\}, \quad$ and $\Delta^{2}=\gamma t / 2$ is, as we will see in the following lines, the effective noise factor. Since $\mathcal{L}\left[\sigma_{+} \sigma_{-}\right]$and $\sigma_{z}$ commute, we can focus 


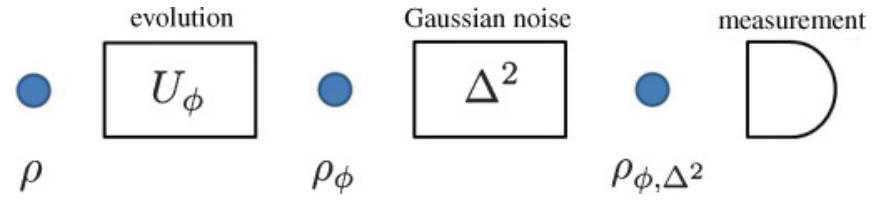

FIG. 1. (Color online) Schematic diagram of the measurement scheme.

on the evolution of $\varrho$, that is, $\dot{\varrho}_{\Delta^{2}}=\gamma \mathcal{L}\left[\sigma_{+} \sigma_{-}\right] \varrho_{\Delta^{2}}$. Upon writing $\varrho_{\Delta^{2}}$ in the eigenbasis of $\sigma_{z}$, the ME leads to differential equations for the matrix elements $\varrho_{n m}(t)=\left\langle n\left|\varrho_{\Delta^{2}}\right| m\right\rangle$, where $\dot{\varrho}_{n m}(t)=-\frac{1}{2} \gamma(n-m)^{2} \varrho_{n m}(t)$, whose solutions read

$$
\varrho_{n m}(t)=e^{-\Delta^{2}(n-m)^{2}} \varrho_{n m}(0),
$$

where $\varrho_{n m}(0)$ are the initial density matrix elements. From Eq. (2) it is clear that, whereas the diagonal elements are left unchanged by the evolution under ME (1), and, in turn, energy is conserved, the off-diagonal ones are progressively destroyed. Finally, the solution of Eq. (1) is $\varrho_{\phi, \Delta^{2}}=U_{\phi} \varrho_{\Delta^{2}} U_{\phi}^{\dagger}$. Since we can consider the noise factor $\Delta^{2}$ as a fixed parameter, in the following we do not write explicitly the dependence on it.

It is worth noting that the same evolution as (2) can be also obtained by the application of a random, zero-mean Gaussiandistributed phase shift to a quantum state. Since the phase shift of an amount $\varphi$ is described by the unitary operator $U_{\varphi} \equiv$ $\exp \left(-i \varphi \sigma_{z}\right)$, we can write the state degraded by the Gaussian phase noise as follows:

$$
\begin{aligned}
\varrho_{\mathrm{Gn}} & =\int_{\mathrm{R}} d \varphi \frac{e^{-\varphi^{2} /\left(4 \Delta^{2}\right)}}{\sqrt{4 \pi \Delta^{2}}} U_{\varphi} \varrho(0) U_{\varphi}^{\dagger} \\
& =\sum_{n m} \int_{\mathrm{R}} d \varphi \frac{e^{-\varphi^{2} /\left(4 \Delta^{2}\right)}}{\sqrt{4 \pi \Delta^{2}}} e^{-i \varphi(n-m)} \varrho_{n m}(0)|n\rangle\langle m| \\
& =\sum_{n m} e^{-\Delta^{2}(n-m)^{2}} \varrho_{n m}(0)|n\rangle\langle m|,
\end{aligned}
$$

which is the same as in Eq. (2). This point will be useful for the experimental demonstration.

The goal of an estimation problem is not only to retrieve the actual value of the unknown parameter (the phase shift in our case) but also to obtain this information with the minimum uncertainty. The ultimate limit to the precision one can reach, that is, the minimum variance, is given by the quantum CramérRao bound [13-16]:

$$
\operatorname{Var}[\phi]=[N H(\phi)]^{-1},
$$

where $N$ is the number of measurements and $H(\phi)$ is the quantum Fisher information (QFI). If we choose a pure probe state, $\varrho=\left|\psi_{0}\right\rangle\left\langle\psi_{0}\right|$, then the QFI equals four times the fluctuation of $\sigma_{z}$; that is, one simply has $H=4\left(1-\left\langle\psi_{0}\left|\sigma_{z}\right| \psi_{0}\right\rangle^{2}\right)$. More generally, for mixed states, $H(\phi)$ can be written as [17]

$$
H(\phi)=2 \sum_{n \neq m} \frac{\left(\lambda_{n}-\lambda_{m}\right)^{2}}{\lambda_{n}+\lambda_{m}}\left|\left\langle\psi_{m}(\phi) \mid \partial_{\phi} \psi_{n}(\phi)\right\rangle\right|^{2},
$$

where the $\left|\psi_{n}(\phi)\right\rangle$ 's are the eigenvectors of the state $\varrho_{\phi}=$ $U_{\phi} \varrho U_{\phi}^{\dagger}$ and $\lambda_{n}$ the corresponding eigenvalues.
The spectral decomposition of $\varrho_{\phi}$ reads as follows

$$
\varrho_{\phi}=\sum_{n} \lambda_{n}\left|\psi_{n}(\phi)\right\rangle\left\langle\psi_{n}(\phi)\left|=\sum_{n} \lambda_{n} U_{\phi}\right| \psi_{n}\right\rangle\left\langle\psi_{n}\right| U_{\phi}^{\dagger},
$$

with $\left|\psi_{n}\right\rangle$ being the eigenvectors of the initial state. If we decompose $\left|\psi_{n}(\phi)\right\rangle$ in the standard basis as follows:

$$
\left|\psi_{n}(\phi)\right\rangle=U_{\phi}\left|\psi_{n}\right\rangle=U_{\phi} \sum_{k} r_{n k}|k\rangle,
$$

then, by substituting (9) into the eigenvalues' equation $\varrho_{\phi}\left|\psi_{n}(\phi)\right\rangle=\lambda_{n}\left|\psi_{n}(\phi)\right\rangle$, after some algebra we obtain

$$
\sum_{k} \varrho_{n k}(0) e^{-\Delta^{2}(n-k)^{2}} r_{q k}=\lambda_{q} r_{q n} \quad \forall n .
$$

Moreover, since $\left|\partial_{\phi} \psi_{n}(\phi)\right\rangle=i \sum_{k} k r_{n k} e^{i k \phi}|k\rangle$, we have [see Eq. (7)]

$$
\left|\left\langle\psi_{m}(\phi) \mid \partial_{\phi} \psi_{n}(\phi)\right\rangle\right|^{2}=\left|\sum_{k} k r_{m k} r_{n k}\right|^{2}
$$

Finally, given $\lambda_{n}$ and $r_{n k}$, we can evaluate the QFI, which is, as expected for a shift parameter, independent of $\phi$.

Generally, the calculation of the eigenvalues $\lambda_{n}$ and the coefficients $r_{n k}$ of the standard basis decomposition (9) is a difficult task, which can be performed by means of numerical analysis. However, in the case of qubit systems, all the calculations can be carried out analytically, as we show in the following.

Upon writing the initial qubit state in the Bloch sphere representation,

$$
\varrho=\frac{1+\boldsymbol{r} \cdot \boldsymbol{\sigma}}{2},
$$

where 1 is the $2 \times 2$ identity matrix, $\boldsymbol{r}=\left(r_{x}, r_{y}, r_{z}\right),|\boldsymbol{r}|^{2} \leqslant 1$, and $\sigma=\left(\sigma_{x}, \sigma_{y}, \sigma_{z}\right)$ is the Pauli matrices vector, then its evolution under the action of the ME (1) can be reduced to the following transformation of the Bloch vector $\boldsymbol{r}$ [18]:

$$
\left(r_{x}, r_{y}, r_{z}\right) \rightarrow\left(r_{x} e^{-\Delta^{2}}, r_{y} e^{-\Delta^{2}}, r_{z}\right),
$$

that is, the Bloch sphere is deformed in such a way that the $z$ component is left unchanged while the $x$ and $y$ components are scaled by the factor $e^{-\Delta^{2}}$ (see Fig. 2). Now, due to symmetry considerations, without lack of generality we focus our attention on the pure state with the Bloch vector:

$$
\boldsymbol{r}=(\sin 2 \theta, 0, \cos 2 \theta),
$$

with $2 \theta$ being the azimuthal angle $(\theta=0$ and $\theta=\pi / 2$ correspond to the north and south poles of the Bloch sphere, respectively).

In the density matrix representation (choosing the $\sigma_{z}$ eigenvectors' basis), after the phase-noise evolution, we have

$$
\varrho=\left(\begin{array}{cc}
\cos ^{2} \theta & e^{-\Delta^{2}} \cos \theta \sin \theta \\
e^{-\Delta^{2}} \cos \theta \sin \theta & \sin ^{2} \theta
\end{array}\right) .
$$

The two eigenvalues are

$$
\lambda_{ \pm}=\frac{1}{2}\left[1 \pm \frac{1+f\left(\theta, \Delta^{2}\right)}{\sqrt{2}}\right]
$$




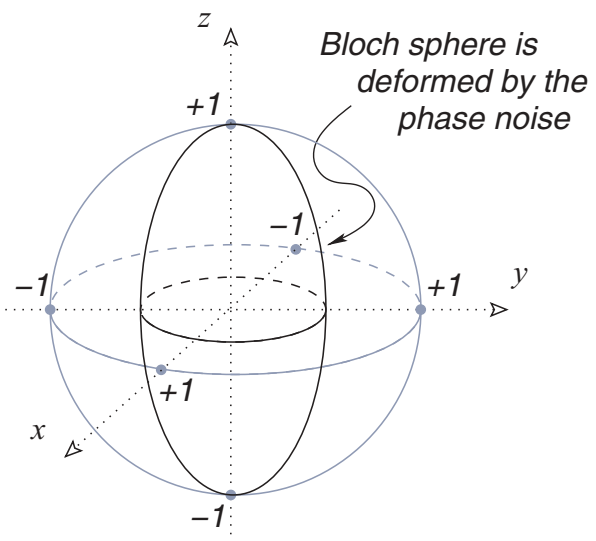

FIG. 2. (Color online) Effect of the phase-destroying ME on the Bloch sphere: the $z$ component is left unchanged while the $x$ and $y$ components are scaled by the factor $e^{-\Delta^{2}}$. See the text for details.

with $f\left(\theta, \Delta^{2}\right)=\sqrt{e^{-2 \Delta^{2}}+\left(1-e^{-2 \Delta^{2}}\right) \cos 4 \theta}$, and the corresponding eigenvectors read

$$
\begin{aligned}
\left|\psi_{ \pm}\right\rangle & =\frac{1}{Z_{ \pm}}\left[\begin{array}{c}
g_{ \pm}\left(\theta, \Delta^{2}\right) \\
1
\end{array}\right], \\
& =\frac{1}{Z_{ \pm}}\left[g_{ \pm}\left(\theta, \Delta^{2}\right)|+1 / 2\rangle+|-1 / 2\rangle\right],
\end{aligned}
$$

$\left\langle\psi_{+} \mid \psi_{-}\right\rangle=0$, and

$$
\begin{aligned}
g_{ \pm}\left(\theta, \Delta^{2}\right) & =\cos 2 \theta \pm f\left(\theta, \Delta^{2}\right) /(\sqrt{2} \sin 2 \theta) \\
Z_{ \pm} & =\sqrt{1+\left[g_{ \pm}\left(\theta, \Delta^{2}\right)\right]^{2}}
\end{aligned}
$$

Substituting the previous equations into Eq. (7) we obtain (remember that for qubit systems $k= \pm 1 / 2$ )

$$
H\left(\theta, \Delta^{2}\right)=e^{-2 \Delta^{2}} \sin ^{2} 2 \theta,
$$

which reaches the maximum for $\theta=\pi / 4$ : the best states for phase estimation also in the presence of phase noise are the equatorial ones, that is, the states laying in the $x-y$ plane of the Bloch sphere. Since the Bures metrics, and then, the Bures distance [19-25] between states, is proportional to the QFI $[17,26]$, this result can be easily understood from a geometrical point of view. If we choose two states, one equatorial and the other not, and shift them by the same amount, $\phi$, then the distance on the Bloch sphere between the initial states and the shifted counterparts is larger for the equatorial states (see Fig. 3) which, in turn, allows better estimation.

The optimal quantum estimator leading to (20) can be written as [17]

$$
O_{\phi}=\phi \mathrm{I}+\frac{L_{\phi}}{H\left(\theta, \Delta^{2}\right)},
$$

where we introduced the symmetric logarithmic derivative

$$
\partial_{\phi} \varrho_{\phi}=\frac{L_{\phi} \varrho_{\phi}+\varrho_{\phi} L_{\phi}}{2},
$$

and $\varrho_{\phi}$ is the state of the qubit initially prepared in a pure state with Bloch vector $\boldsymbol{r}$ given by (13), shifted by the application of the unitary transformation $U_{\phi}=\exp \left(-i \phi \sigma_{+} \sigma_{-}\right)=$ $\exp \left[-\frac{i}{2} \phi\left(\sigma_{z}+\mathrm{I}\right)\right]$ and degraded by the evolution through the

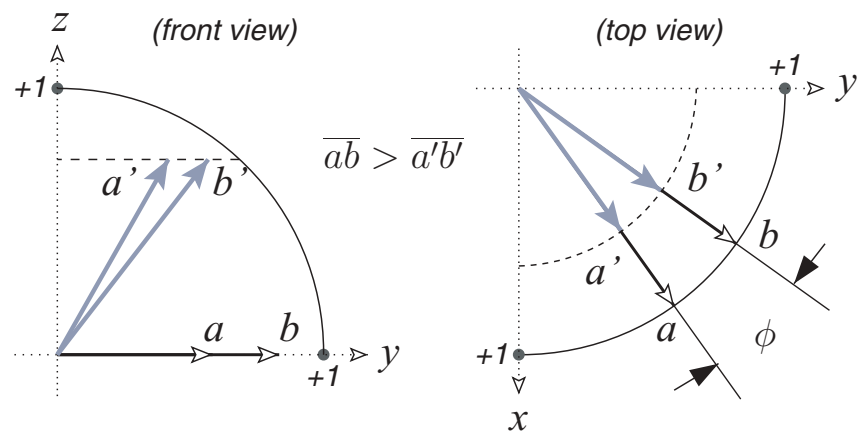

FIG. 3. (Color online) The distance $\overline{a b}$ on the Bloch sphere between two equatorial states separated by a phase shift, $\phi$, is larger than the distance $\overline{a^{\prime} b^{\prime}}$ between nonequatorial states separated by the same amount, $\phi$. The same conclusion holds in the presence of phase noise: best estimation is achieved by involving equatorial states.

noisy environment:

$$
\varrho_{\phi}=\left(\begin{array}{cc}
\cos ^{2} \theta & e^{-i \phi-\Delta^{2}} \cos \theta \sin \theta \\
e^{i \phi-\Delta^{2}} \cos \theta \sin \theta & \sin ^{2} \theta
\end{array}\right) .
$$

One finds

$L_{\phi}=i \frac{2 g_{+} g_{-}\left(g_{+}-g_{-}\right)\left(\lambda_{-}-\lambda_{+}\right)}{Z_{+}^{2} Z_{-}^{2}\left(\lambda_{+}+\lambda_{-}\right)}\left(\sigma_{+} e^{i \phi}-\sigma_{-} e^{-i \phi}\right)$,

where $\lambda_{ \pm}, g_{ \pm} \equiv g_{ \pm}\left(\theta, \Delta^{2}\right)$ and $Z_{ \pm}$are given in (16) and (19), respectively. If we choose $\theta=\pi / 4$, Eq. (24) reduces to

$$
L_{\phi}=i e^{-\Delta^{2}}\left(\sigma_{+} e^{i \phi}-\sigma_{-} e^{-i \phi}\right) .
$$

\section{PHASE-SHIFT ESTIMATION BY SPIN MEASUREMENT}

Let us now consider a realistic scenario where, in order to estimate $\phi$, we measure the spin in a generic direction in the plane, that is, the observable

$$
\Theta_{\alpha}=\sigma_{x} \cos \alpha+\sigma_{y} \sin \alpha,
$$

whose eigenvectors $\left|\Sigma_{ \pm}(\alpha)\right\rangle, \Theta_{\alpha}\left|\Sigma_{ \pm}(\alpha)\right\rangle= \pm\left|\Sigma_{ \pm}(\alpha)\right\rangle$ are

$$
\left|\Sigma_{ \pm}(\alpha)\right\rangle=\frac{1}{\sqrt{2}}\left(e^{-i \alpha}|+1 / 2\rangle \pm|-1 / 2\rangle\right) .
$$

For the qubit of Eq. (23), the probabilities to obtain the outcomes \pm 1 given the phase shift $\phi$ read

$$
\begin{aligned}
P_{\Delta^{2}}( \pm 1 \mid \phi) & =\operatorname{Tr}\left[\left|\Sigma_{ \pm}(\alpha)\right\rangle\left\langle\Sigma_{ \pm}(\alpha)\right| \varrho_{\phi}\right] \\
& =\frac{1}{2}\left[1 \pm e^{-\Delta^{2}} \cos (\alpha-\phi) \sin 2 \theta\right],
\end{aligned}
$$

and the expectation value is

$$
\left\langle\Theta_{\alpha}\right\rangle=\operatorname{Tr}\left[\Theta_{\alpha} \varrho_{\phi}\right]=e^{-\Delta^{2}} \cos (\alpha-\phi) \sin 2 \theta .
$$

The corresponding Fisher information turns out to be

$$
\begin{aligned}
F\left(\phi, \Delta^{2}\right) & =\sum_{k= \pm 1} P_{\Delta^{2}}(k \mid \phi)\left[\partial_{\phi} \ln P_{\Delta^{2}}(k \mid \phi)\right]^{2} \\
& =\frac{e^{-2 \Delta^{2}} \sin ^{2}(\alpha-\phi) \sin ^{2} 2 \theta}{1-e^{-2 \Delta^{2}} \cos ^{2}(\alpha-\phi) \sin ^{2} 2 \theta},
\end{aligned}
$$




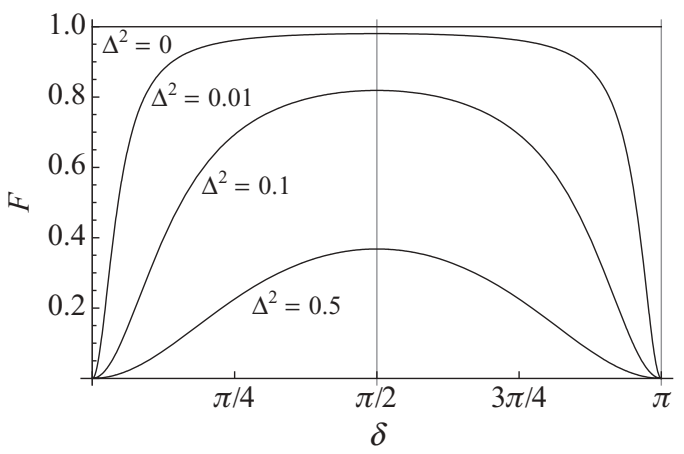

FIG. 4. Plot of the Fisher information $F\left(\phi, \Delta^{2}\right)$ in the case of equatorial qubit probe states $(\theta=\pi / 4)$ as a function of $\delta=\alpha-\phi$ and different values of $\Delta^{2}$.

which is plotted in Fig. 4 in the case of equatorial probe states $(\theta=\pi / 4)$ as a function of $\delta=\alpha-\phi$ and different values of $\Delta^{2}$. As we can see, in the absence of noise $\left(\Delta^{2}=0\right)$ one has $F=1, \forall \alpha, \phi$, that is, the Fisher information is equal to the QFI $H$ in Eq. (20) [27,28]. When noise affects the propagation, the maximum of $F$, which corresponds to the QFI $H$, is achieved for $\delta=\alpha-\phi=\pi / 2$, whereas it goes to zero as $\alpha-\phi=k \pi$, $k \in \mathrm{N}$. These results can be better understood considering the sensitivity of the measurement (actually this is the square of the sensitivity):

$$
\mathcal{S}=\frac{\operatorname{Var}\left[\Theta_{\alpha}\right]}{\left(\partial_{\phi}\left\langle\Theta_{\alpha}\right\rangle\right)^{2}}=\frac{1-\left\langle\Theta_{\alpha}\right\rangle^{2}}{\left(\partial_{\phi}\left\langle\Theta_{\alpha}\right\rangle\right)^{2}},
$$

that is, the ratio between the fluctuations of $\left\langle\Theta_{\alpha}\right\rangle$ and how $\left\langle\Theta_{\alpha}\right\rangle$ varies with respect to $\phi ; \sqrt{\mathcal{S}}$ represents the smallest change of $\phi$ that can be detected with our measurement (up to the statistical scaling, of course). In the present case,

$$
\mathcal{S}\left(\phi, \Delta^{2}\right)=\frac{1-e^{-2 \Delta^{2}} \cos ^{2}(\alpha-\phi) \sin ^{2} 2 \theta}{e^{-2 \Delta^{2}} \sin ^{2}(\alpha-\phi) \sin ^{2} 2 \theta},
$$

which is just the inverse of Eq. (32): the maximum of $F$ (maximum information) corresponds to the case of maximum sensitivity (minimum of $\mathcal{S}$ ). If $\Delta^{2}=0$ one finds that $\operatorname{Var}\left[\Theta_{\alpha}\right]$ and $\left(\partial_{\phi}\left\langle\Theta_{\alpha}\right\rangle\right)^{2}$ are always equal, no matter the values of $\alpha$ and $\phi$. When noise is acting, the maximum of $F$ at $\delta=\pi / 2$ corresponds to the minimum of $\mathcal{S}\left(\phi, \Delta^{2}\right)$; this fact can be also understood by geometrical means addressing the special case of $\alpha=0\left(\Theta_{0}=\sigma_{x}\right)$. In this case the result of the measurement carried out on the probe is just the projection onto the $x$ axis: for a fixed change $d \phi$, the change of $\left\langle\Theta_{0}\right\rangle$ at $\phi=0$ is smaller than the one at $\phi=\pi / 2$ (see Fig. 5).

The Fisher information depends on the actual, unknown value of $\phi$. However, we can perform an adaptive, two-step method to achieve the QFI. During the first step, we use a small amount of data to obtain a rough estimate, $\tilde{\phi}$, of the phase shift; then, at the second step, we tune $\Theta_{\alpha}$ according to the transformation $\alpha \rightarrow \tilde{\phi}+\pi / 2$. The (eventual) repetition of these two steps allows the QFI limit to be reached. The same result can be obtained by fixing the measurement at a chosen $\alpha$ and tuning the probe state by applying a suitable rotation.

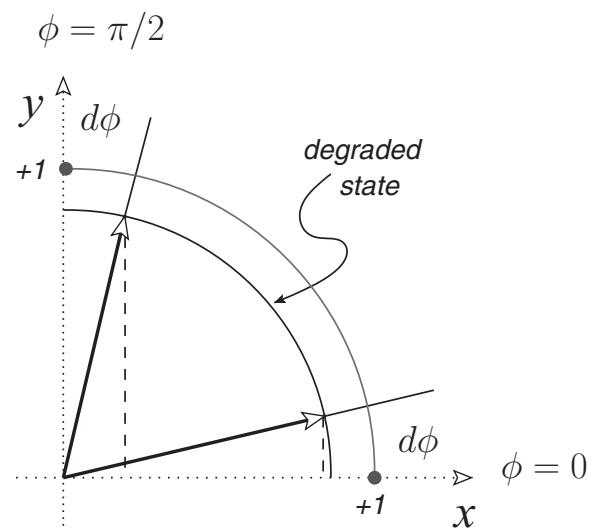

FIG. 5. Sensitivity of $\sigma_{x}$ measurement to a small change $d \phi$ in the phase shift. The projections onto the $x$ axis are the expectations $\left\langle\Theta_{0}\right\rangle$, with $\Theta_{0}=\sigma_{x}$. For a fixed change $d \phi$, the change of $\left\langle\Theta_{0}\right\rangle$ at $\phi=0$ is smaller than the one at $\phi=\pi / 2$. For the sake of simplicity we sketch only the upper right quarter of the Bloch sphere $x-y$ equatorial plane.

\section{PHASE-SHIFT ESTIMATION FOR POLARIZATION ENCODED OPTICAL QUBITS}

In our proposal the qubit state corresponds to the polarization degree of freedom of a coherent state. We refer to $|H\rangle$ and $|V\rangle$, respectively, as horizontal and vertical polarization. Initially, we set the polarization at $|+\rangle=\frac{1}{\sqrt{2}}(|H\rangle+|V\rangle)$ (equatorial state) and apply the phase shift with eventual Gaussian noise (as we have shown in Sec. II, this is equivalent to a nondissipative phase noise). Then we measure $\sigma_{x}$, which corresponds to inserting a half-wave plate (HWP) at $22.5^{\circ}$ in front of a polarizing beam splitter (PBS) and recording the number of counts $n_{+}$and $n_{-}$at the two outputs, which correspond to the outcomes +1 and -1 , respectively. Once a data sample $X$ has been acquired, one needs to give an estimation of the phase shift. Here we consider two strategies: one based on the inversion of the probability functions, whose uncertainties come from the error propagation; the other applies the Bayes theorem to retrieve a prior distribution of phase shift and knowing this distribution we can calculate all the desired moments.

\section{A. Estimation by inversion}

If we send $M$ copies of our state, then we can write

$$
P_{\Delta^{2}}( \pm 1 \mid \phi)=\frac{n_{ \pm}}{n_{+}+n_{-}},
$$

and, by inverting Eq. (29), after some algebra we obtain the following expression for the phase-shift estimator:

$$
\phi_{\mathrm{inv}}=\arccos \left(\frac{n_{+}-n_{-}}{n_{+}+n_{-}} e^{\Delta^{2}}\right) .
$$

The uncertainty in the estimation is thus given by

$$
\operatorname{Var}\left[\phi_{\text {inv }}\right]=\left(\frac{\partial \phi_{\text {inv }}}{\partial n_{+}}\right)^{2} \sigma^{2}\left(n_{+}\right)+\left(\frac{\partial \phi_{\text {inv }}}{\partial n_{-}}\right)^{2} \sigma^{2}\left(n_{-}\right),
$$

where $\sigma^{2}\left(n_{ \pm}\right)$are the fluctuations of the numbers of outcomes $n_{ \pm}$. It is worth noting that at each shot, that is, for each sent 
copy of the coherent state, the detected number of photons fluctuates according to the laws of quantum mechanics.

\section{B. Bayesian estimation}

Given the data sample $X=\left\{x_{1}, x_{2}, \ldots, x_{N}\right\}$, where $x_{k} \in$ $\{-1,+1\}, \forall k$, we can define the sample probability:

$$
P(X \mid \phi)=\prod_{k=1}^{N} P_{\Delta^{2}}\left(x_{k} \mid \phi\right)
$$

that is, the probability to obtain the whole data sample $X$ given the unknown phase $\phi$. By means of the Bayes theorem one can write the a posteriori probability [29]

$$
P(\phi \mid X)=\frac{1}{\mathcal{N}} \prod_{k=1}^{N} P_{\Delta^{2}}\left(x_{k} \mid \phi\right), \quad \mathcal{N}=\int_{\Phi} d \phi P(\phi \mid X),
$$

where $\Phi$ is the parameter space. The probability (39) is the probability distribution of $\phi$ given the data sample $X$. The Bayesian estimator are thus obtained as

$$
\begin{aligned}
\phi_{\mathrm{B}} & =\int_{\Phi} d \phi \phi P(\phi \mid X), \\
\operatorname{Var}\left[\phi_{\mathrm{B}}\right] & =\int_{\Phi} d \phi\left(\phi-\phi_{\mathrm{B}}\right)^{2} P(\phi \mid X) .
\end{aligned}
$$

Bayesian estimator are known to be asymptotically optimal; namely, they allow one to achieve the Cramér-Rao bound as the size of the data sample increases [17,29-32].

\section{Monte Carlo simulated experiments}

Figure 6 shows the variances of the estimated phases from Monte Carlo simulated experiments as a function of the (a)

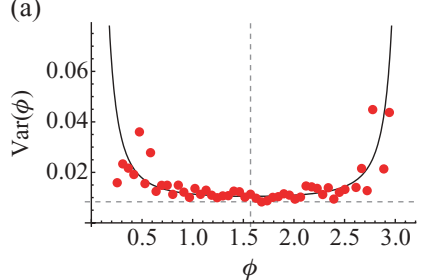

(c)

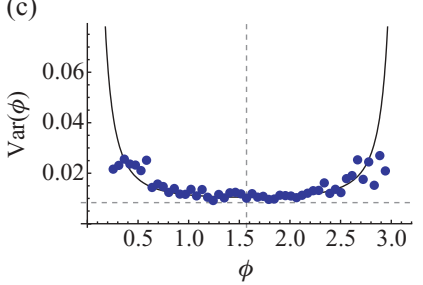

(b)

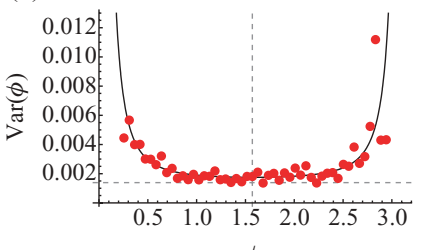

$\phi$

(d)

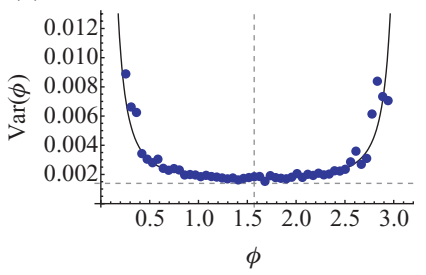

FIG. 6. (Color online) Variances from simulated Monte Carlo experiments performed with $M=60$ copies of the input state and $\Delta=0.34$. The plots on the top refer to the inversion method, the bottom ones to Bayesian analysis. For the plots on the left we chose a coherent state with an average number of photons equal to $\bar{n}=2$, for those on the right we set $\bar{n}=12$. The solid line is the CramérRao bound $1 /(F M \bar{n})$. The dashed horizontal line is the quantum Cramér-Rao bound $1 /(H M \bar{n})$. Notice the asymptotic optimality of the Bayesian estimation. unknown phase shift. The simulations have been performed by sending $M=60$ copies of the input coherent state and choosing $\Delta=0.34$. The plots on the top refer to the inversion method; the plots on the bottom refer to Bayesian analysis. We investigated two different cases: low energy (average number of photons equal to $\bar{n}=2$, left plots) and high energy ( $\bar{n}=12$, rights plots). The solid line is the Cramér-Rao bound obtained from Eq. (34) and the dashed horizontal line is the quantum Cramér-Rao bound; both are rescaled by the effective number of measurements $N=M \bar{n}$. We can see that in the low-energy regime there is a more evident deviation from the expected behavior (solid line) at the extremes of the phase interval $(0, \pi)$. This is the manifestation of a systematic error due to phase window sampling; that is, the tails of the Gaussian distributions, simulating the phase-noise, are truncated at the boundary and refolded inside the interval. Notice the asymptotic optimality of the Bayesian estimation when the energy and, thus, the number of events increase. It is also useful to observe how the fluctuations of the variances obtained by using the Bayesian method are less than the other ones, which actually depend on the fluctuations of the average number of photons. A further investigation of Fig. 6 leads us to conclude that both the methods are robust over a large interval of phases: for the considered examples the variance is almost constant over the phase interval $(0.5,2.5)$.

\section{EXPERIMENTAL SETUP AND RESULTS}

The experimental demonstration of our scheme is based on a potassium dihydrogen phosphate (KDP) crystal that allows both the manipulation of the optical qubit polarization and the simulation of a phase diffusion environment. In Fig. 7 we sketch the experimental setup.

A linearly polarized He:Ne laser (Thorlabs HRP120) generates coherent states that are prepared in the initial qubit

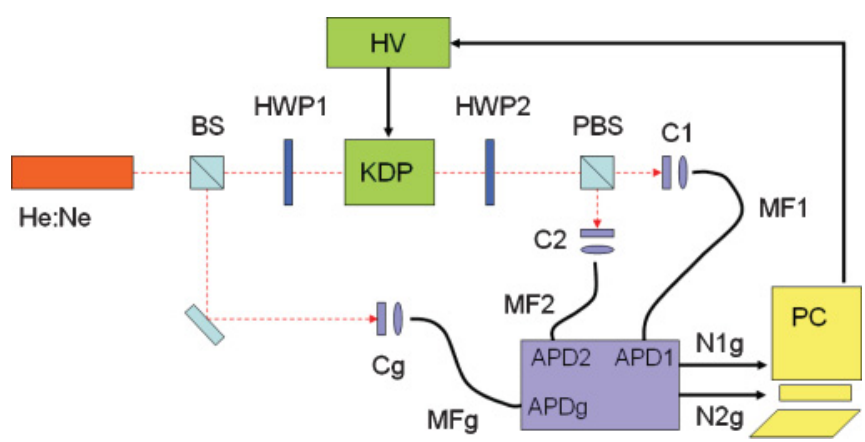

FIG. 7. (Color online) Sketch of the experimental setup to estimate the phase of a polarization qubit in the presence of nondissipative noise. The qubit state is set to $|+\rangle$ by inserting a half-wave plate (HWP1) along the path of the He:Ne laser output, while a KDP crystal, driven by a high-voltage generator (HV), is used both to add the phase shift $\phi$ and to simulate the noise. A second half-wave plate at $22.5^{\circ}$ (HWP2) together with a polarizing beam splitter (PBS) implements the measurement (the couplers, $\mathrm{C}$, and the multimode fibers, $\mathrm{MF}$, are used to bring the signals to the avalanche photodiodes, APD). Finally, the contemporary counts $N_{1 g}$ and $N_{2 g}$ coming from the APD1 and APD2 and from the APDg, that is, the gate channel signal coming from the beam splitter (BS) placed in front the laser source, are acquired by a PC module. 
(a)

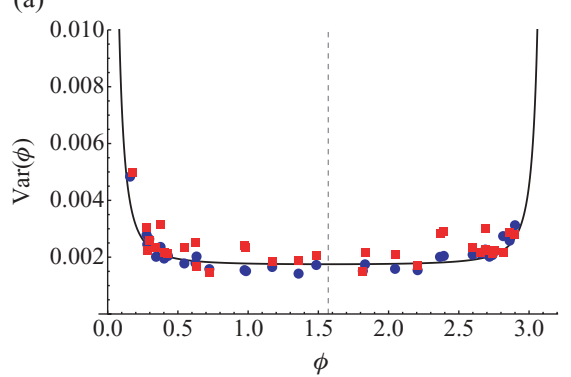

(b)

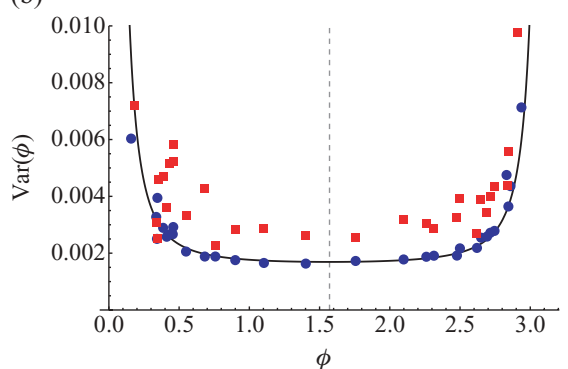

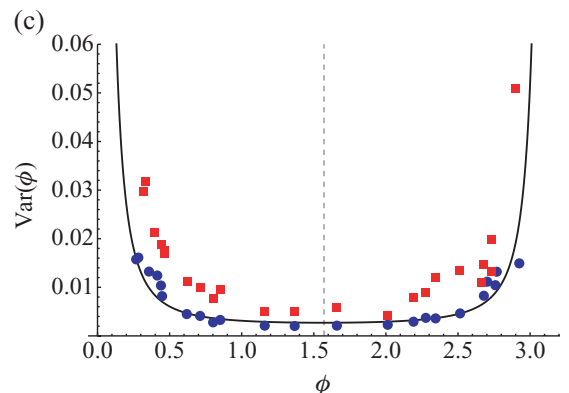

FIG. 8. (Color online) Variances from experiments performed with $M=60$ copies of the input state and (a) $\Delta=0.13 \pm 0.02$, (b) $\Delta=0.24 \pm 0.03$, and (c) $\Delta=0.48 \pm 0.05$. Red squares refer to inversion method; blue circles refer to Bayesian analysis. We also have (a) $\bar{n}=9.83 \pm 0.93$, (b) $\bar{n}=11.06 \pm 0.43$, and (c) $\bar{n}=9.78 \pm 0.95$. The solid line is the Cramér-Rao bound $1 /(F M \bar{n})$.

state $|+\rangle=\frac{1}{\sqrt{2}}(|H\rangle+|V\rangle)$ by means of a first half-wave plate (HWP1). Then the qubits pass through a KDP crystal driven by a stabilized high-voltage generator (HV), up to a maximum of $6 \mathrm{kV}$. Through the application of an electrostatic field, $E_{z}$, along the $z$ axis of the KDP (which is also the direction of propagation of the qubit), a phase shift, $\phi\left(E_{z}\right)$, is introduced between the qubit polarization components along axes $x$ and $y$. The crystal is oriented with the $x$ axis parallel to the horizontal polarization and tilted around the vertical axis $y$ in order to avoid the multiple internal reflections superposed with the main beam, which are removed by a pinhole.

The detection system consists of a second half-wave plate (HWP2), a PBS, absorption filters, and three detectors. The HWP2 plate is set at $22.5^{\circ}$ with respect to the horizontal axis and the PBS selects the polarization. Light signals are focused into multimode fibers (MF) and sent to the detectors APD1 and APD2. The detectors are single-photon counting modules (SPCM) based on avalanche photodiodes operated in Geiger mode with passive quenching. For the coincidence counts an electronic circuit based on AND gates is used. A 50:50 beam splitter (BS) is placed before the HWP1 plate to add a gate channel $(\mathrm{g})$ for the counting measurement. The aim is to make acquisition with a low number of counts maintaining a high signal-to-noise ratio, $N_{i} / N_{i, \mathrm{dc}}(i=1,2)$. This is the reason for the coincidence counting, according to the formula $(i=1,2)$

$$
\begin{aligned}
N_{i g} & =\left(N_{i}+N_{i, \mathrm{dc}}\right) N_{g} \Delta t=N_{i} N_{g} \Delta t+N_{i, \mathrm{dc}} N_{g} \Delta t \\
& =N_{i g, \text { true }}+N_{i g, \mathrm{dc}},
\end{aligned}
$$

where $N_{i}$ and $N_{g}$ are, respectively, the direct counts on the detectors $\mathrm{APD}_{i}$ and $\mathrm{APD}_{g}, N_{i g}$ are the coincidence counts $N_{i g, \text { dc }}$ and $N_{i, \mathrm{dc}}$ are, respectively, the coincidence and direct dark counts; and $\Delta t$ is the coincidence time window of the electronic counting module $(\Delta t=90 \mathrm{~ns}$ ). Upon selecting the time window of the acquisition, $N_{i g}$ may set as low as we want, while keeping the ratio $N_{i g \text {,true }} / N_{i g, \mathrm{dc}}=N_{i} / N_{i, \mathrm{dc}}$ constant. We measure about $10^{5}$ counts/s on the detector APDg and a maximum of about $9 \times 10^{4}$ counts/s on the other two but we can adjust these direct counts by changing filters or the detector voltages above breakdown. The dark counts are below 200 counts/s. Each acquisition is taken with a 10 -ms time window in order to have $\bar{n} \simeq 10$ in the contemporary counts. Note that the signal-to-noise ratio remains $\simeq 10^{3}$. The rising and falling times of the high-voltage generator are measured at around $200 \mathrm{~ms}$; therefore the waiting time between two subsequent measurements is set at $240 \mathrm{~ms}$.

We made $M=60$ acquisitions for each phase shift $\phi$. Notice that each acquisition corresponds to a different random phase distributed according to a Gaussian distribution centered on $\phi$ and with standard deviation $\sigma^{2}(\phi)=\Delta^{2} / 2$ [see Eq. (3)]. In our implementation for each acquisition we send a different voltage to the KDP crystal according to a proper calibration curve, $\phi(V)$. The results are shown in Fig. 8, where we plot the theoretical Cramér-Rao bound (solid line) and the experimental variances obtained using the inversion method (red squares) and the Bayesian analysis (blue circles). The Cramér-Rao bound is quickly reached by Bayesian estimation, even for a relatively small number of measurements $(N=M \bar{n}$ in the figure, with $M=60$ and $\bar{n} \simeq 10$ ).

\section{A. Adaptive method for optimality}

As we have seen, Bayesian estimation allows us to reach the Cramér-Rao bound for any orientation of the measurement angle. On the other hand, as we pointed out in Sec. III, optimal estimation, that is, with precision at quantum Cramér-Rao limit, is achieved only if $\phi=\pi / 2$. In this section we show that ultimate precision can be always achieved upon the application of an adaptive method [32-34]. In the present case, we proceed as follows: (i) we start our estimation by obtaining a first value $\phi_{1}$ of the unknown phase; (ii) we set $\alpha$ of the measured observable (26) to the value $\alpha_{1}=\pi / 2+\phi_{1}$ (this can be also

TABLE I. Phase estimation using the adaptive method (see the text for details). The actual value of the phase is $\phi=0.17 \pm 0.01$. The amplitude of the phase noise is $\Delta=0.46 \pm 0.06$ and the energy of the coherent state is $\bar{n}=10.97 \pm 0.67$. For each step we used $M=55$ repetitions for the estimation. For these values of the parameters the quantum Cramér-Rao bound, evaluated from Eq. (20) and rescaled by the number of measurments $N=M \bar{n}$, is equal to $\operatorname{Var}(\phi) \simeq 2.52 \times 10^{-3}$.

\begin{tabular}{lcc}
\hline \hline Step & $\operatorname{Var}(\phi)$ & $\phi^{\text {(est) }}$ \\
\hline 1 & $4.21 \times 10^{-3}$ & $0.33 \pm 0.06$ \\
2 & $2.49 \times 10^{-3}$ & $0.22 \pm 0.05$ \\
3 & $2.52 \times 10^{-3}$ & $0.19 \pm 0.05$ \\
4 & $2.59 \times 10^{-3}$ & $0.20 \pm 0.05$ \\
\hline \hline
\end{tabular}


obtained by adding the phase shift $\alpha_{1}^{\prime}=\pi / 2-\phi_{1}$ to the qubit itself), so the new phase is near to $\pi / 2$. One can also repeat this procedure many times in order to refine the estimation. However, as one can see in Figs. 6 and 8, the optimal condition is almost achieved after the first step and the variance is nearly constant around the optimality region. We report the results in Table I, where one can read the step number of the adaptive method, the corresponding variance, and the estimated phase. Note that already at the second step optimal estimation has been achieved; that is, the experimental variances are close to the optimal variance given by the quantum Cramér-Rao bound.

\section{CONCLUSIONS}

We have demonstrated an optimal estimation scheme for the phase shift imposed to a polarization encoded optical qubit in the presence of phase diffusion. Our scheme is based on polarization measurement assisted by Bayesian estimation and allows the ultimate quantum bound to precision to be achieved using a limited number of measurements. In turn, the Bayes estimator is known to be asymptotically unbiased, but for practical implementation is of interest to evaluate quantitatively how many measurements are needed to achieve the asymptotic region. Our results indicate that Bayesian inference represents a useful tool for phase estimation. An adaptive method to achieve optimal estimation in any working regime, that is, for any value of the unknown phase-shift, has been also analyzed in detail and experimentally demonstrated. As a future perspective we foresee the possibility to investigate the effects of different kind of noises and to employ entangled probes to increase the overall stability of the estimation procedure, a results which has been theoretically established for the ideal (i.e., without noise) case [29].

\section{ACKNOWLEDGMENTS}

SO and MGG thank A. Smerzi and P. Hyllus for discussions. This work has been partially supported by the CNR-CNISM convention.
[1] A. M. Childs, I. L. Chuang, and D. W. Leung, Phys. Rev. A 64, 012314 (2001).

[2] M. W. Mitchell, C. W. Ellenor, S. Schneider, and A. M. Steinberg, Phys. Rev. Lett. 91, 120402 (2003).

[3] G. M. D’Ariano and P. Lo Presti, Phys. Rev. Lett. 91, 047902 (2003); G. M. D’Ariano et al., J. Phys. A 34, 93 (2001).

[4] J. L. O'Brien, G. J. Pryde, A. Gilchrist, D. F. V. James, N. K. Langford, T. C. Ralph, and A. G. White, Phys. Rev. Lett. 93, 080502 (2004).

[5] S. H. Myrskog, J. K. Fox, M. W. Mitchell, and A. M. Steinberg, Phys. Rev. A 72, 013615 (2005).

[6] M. G. A. Paris and J. Rehacek (Eds.), Quantum State Estimation (Springer, New York, 2004), Lecture Notes in Physics, Vol. 649.

[7] G. M. D'Ariano et al., J. Phys. A 34, 93 (2001).

[8] K. Banaszek, G. M. D’Ariano, M. G. A. Paris, and M. F. Sacchi Phys. Rev. A 61, 010304(R) (1999).

[9] D. F. V. James, P. G. Kwiat, W. J. Munro, and A. G. White, Phys. Rev. A 64, 052312 (2001).

[10] Z. Hradil, Phys. Rev. A 55, R1561 (1997).

[11] J. H. Cole, S. G. Schirmer, A. D. Greentree, C. J. Wellard, D. K. L. Oi, and L. C. L. Hollenberg, Phys. Rev. A 71, 062312 (2005).

[12] J. H. Cole, A. D. Greentree, D. K. L. Oi, S. G. Schirmer, C. J. Wellard, and L. C. L. Hollenberg, Phys. Rev. A 73, 062333 (2006).

[13] H. P. Yuen and M. Lax, IEEE Trans. Inf. Theory 19, 740 (1973).

[14] C. W. Helstrom and R. S. Kennedy, IEEE Trans. Inf. Theory 20, 16 (1974).

[15] S. L. Braunstein and C. M. Caves, Phys. Rev. Lett. 72, 3439 (1994).
[16] S. Braunstein, C. Caves, and G. Milburn, Ann. Phys. 247, 135 (1996).

[17] M. G. A. Paris, Int. J. Quantum Inf.7, 125 (2009).

[18] N. A. Nielsen and I. L. Chuang, Quantum Computation and Quantum Information (Cambridge University Press, Cambridge, UK, 2000).

[19] D. J. C. Bures, Trans. Am. Math. Phys. 135, 199 (1969).

[20] A. Uhlmann, Rep. Math. Phys. 9, 273 (1976).

[21] M. Hübner, Phys. Lett. A163, 239 (1992).

[22] R. Josza, J. Mod. Opt. 41, 2315 (1994).

[23] P. B. Slater, J. Phys. A 29, L271 (1996); Phys. Lett. A244, 35 (1998).

[24] M. J. W. Hall, Phys. Lett. A242, 123 (1998).

[25] J. Dittmann, J. Phys. A 32, 2663 (1999).

[26] H.-J. Sommers et al., J. Phys. A 36, 10083 (2003).

[27] B. Yurke, S. L. McCall, and J. R. Klauder, Phys. Rev. A 33, 4033 (1986).

[28] L. Pezzé, A. Smerzi, G. Khoury, J. F. Hodelin, and D. Bouwmeester, Phys. Rev. Lett. 99, 223602 (2007).

[29] B. Teklu et al., J. Phys. B 42, 035502 (2009).

[30] Z. Hradil, Phys. Rev. A 51, 1870 (1995).

[31] Z. Hradil, R. Myska, J. Perina, M. Zawisky, Y. Hasegawa, and H. Rauch, Phys. Rev. Lett. 76, 4295 (1996).

[32] S. Olivares and M. G. A. Paris, J. Phys. B 42, 055506 (2009).

[33] R. D. Gill and S. Massar, Phys. Rev. A 61, 042312 (2000).

[34] M. Hayashi and K. Matsumoto, Asymptotic Theory of Quantum Statistical Inference: Selected Papers, edited by M. Hayashi (World Scientific, Singapore, 2005), Chap. 1, p. 162; arXiv:quant-ph/0308150. 